\title{
Editors' Introduction: Internet-of-Things and Big Data for Smarter Healthcare: From Device to Architecture, Applications and Analytics
}

\author{
Farshad Firouzi* $^{*}$ Amir M. Rahmani ${ }^{\dagger}$, K. Mankodiya ${ }^{\ddagger}$, M. Badaroglu ${ }^{\S}$, G. V. Merrett ${ }^{\Uparrow}$, P. Wong $\|$, Bahar Farahani** \\ ${ }^{*}$ MSG Group, Germany \\ ${ }^{\dagger}$ University of California, Irvine, USA \& TU Wien, Vienna, Austria \\ $\ddagger$ University of Rhode Island, USA \\ $\S$ Qualcomm Technologies, Belgium \\ IUniversity of Southampton, UK \\ "Stanford University, USA \\ ** Department of Electrical and Computer Engineering, University of Tehran, Iran
}

\begin{abstract}
The technology and healthcare industries have been deeply intertwined for quite some time. New opportunities, however, are now arising as a result of fast-paced expansion in the areas of the Internet of Things (IoT) and Big Data. In addition, as people across the globe have begun to adopt wearable biosensors, new applications for individualized eHealth and mHealth technologies have emerged. The upsides of these technologies are clear: they are highly available, easily accessible, and simple to personalize; additionally they make it easy for providers to deliver individualized content cost-effectively, at scale. At the same time, a number of hurdles currently stand in the way of truly reliable, adaptive, safe and efficient personal healthcare devices. Major technological milestones will need to be reached in order to address and overcome those hurdles; and that will require closer collaboration between hardware and software developers and medical personnel such as physicians, nurses, and healthcare workers. The purpose of this special issue is to analyze the top concerns in IoT technologies that pertain to smart sensors for health care applications; particularly applications targeted at individualized tele-health interventions with the goal of enabling healthier ways of life. These applications include wearable and body sensors, advanced pervasive healthcare systems, and the Big Data analytics required to inform these devices.
\end{abstract}

\section{INTRODUCTION}

In the recent years, the synergy between the healthcare and technology has taken a big leap across the world. For example, Internet of Things (IoT) and Big Data Analytics are increasingly gaining popularity for the next generation of eHealth and mHealth services. While these fields are emerging, they also bring new challenges, especially when the target is healthcare that itself is a complicated system, demanding consistent, suitable, safe, flexible, and energy-efficient solutions.

IoT in healthcare covering the markets of medical devices, systems, software, and services is expected to grow to a market size of $\$ 300 B$ by 2022 according to the market analyst, Grand View Research, as shown in Fig. 1. Government initiatives are also likely to promote this demand for those personalized ehealthcare.

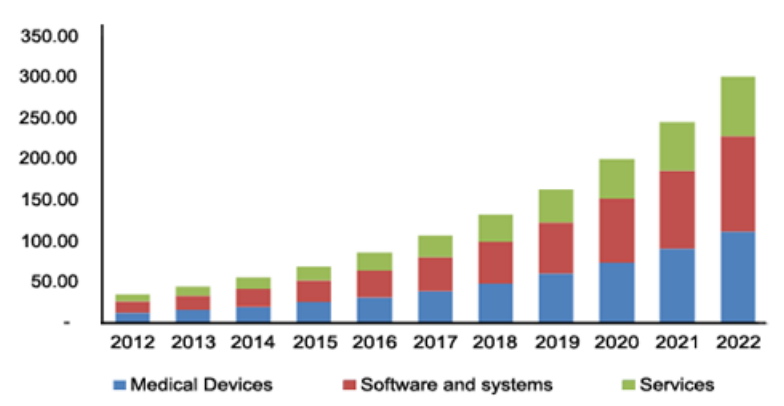

Fig. 1: North America IoT in Healthcare Market Growth, by Component, 2012 - 2022 (USD Billion) [1].

Fig. 2 outlines the general architectural elements required for healthcare IoT systems (Health-IoT), which includes three main components [2]: i) body area sensor network, ii) Internetconnected smart gateways, also known as Fog layer [3], or a local access network, and iii) cloud and big data support. Various applications provide services to different stakeholders in the system through this platform. Data generated from sensors attached to users is made available to doctors, family and authorized parties giving them the ability to check the subjects vital signs from anywhere at any time as well as performing intelligent decision making to assist healthcare workers [4], [5]. In this generic architecture, Smart e-Health Gateways [6], [7], which support different communication protocols, act as the bridging point between a sensor network and the local switch/Internet. They receive data from different subnetworks, perform protocol conversion, and provide other higher level services such as data aggregation, mining, filtering, encryption, local notification, and dimensionality reduction [3], [8], [9]. The back-end of the system resided at the cloud layer consists of two main parts: 1) A cloud-based back-end infrastructure 
including data storage, data analytics, decision making, etc, 2) User interface which acts as a dashboard for medical caregivers and performs user control, and data visualization and apprehension. Using our advanced inference algorithms and machine learning techniques, the health IoT system automatically learns from sensor measurements and patient history to provide feedback about the current and predicted future health of the patient, and can even raise alarms if necessary.

In such a system, patients behavioural and health related information is recorded by different sensors (e.g., body-worn or implanted sensors) with which the patient is equipped for personal monitoring of multiple parameters. This health data is supplemented with environmental information (e.g., date, time, humidity, temperature). Environment-awareness enables identification of unusual patterns and makes more precise inferences about the situation. Other sensors and actuators (e.g., medical equipment) can be also connected to the systems to transmit data to the medical staff such as video and realtime physiological data.

To support and enable this transformation, this special issue invited the hardware and software communities to come together to present their integrative research in various topics including big data analytics, wearable sensors, fog computing, edge cloud, body sensor networks, and case studies. After review, this special issue accepted six high-quality research articles encompassing novel IoT technologies for smart healthcare including wearable sensors, unobtrusive sensors, body sensor networks, fog computing, RFID, energy efficiency, workload balancing, and reliability that are tailored to support remote interventions to individuals for healthier lifestyles.

\section{A BRIEF REVIEW OF ACCEPTED ARTICLES OF THIS SPECIAL ISSUE}

In this special issue, the Guest Editors have put together some of the new developments and trends in the context of IoT eHealth. We received twenty seven submissions and a total of six papers were accepted. The high level of competition has led to the selection of top-level contributions covering a wide spectrum of topics in the domain of IoT eHealth.

Pagan et al. [10], in their article entitled "Power transmission and workload balancing policies in eHealth mobile cloud computing scenarios", aim at addressing challenges in data acquisition and processing in ambulatory environments. In particular, they study an energetically-efficient massive deployment of an ambulatory body area network for the prediction of migraine events on patients across Europe. They show the effectiveness of techniques such us on-node signal processing and radio policies to make sensor nodes more autonomous and energy-efficient, while proposing workload balancing policies to reduce the computational load and energy consumption in health data centers.

In the article entitled "CUIDATS: An RFIDWSN hybrid monitoring system for smart health care environments," Adame et al. [11] present an IoT hybrid monitoring system which integrates RFID and WSN technologies to track the location of healthcare assets (using passive and active RFID tags), and the location and health of patients (using an active wristband monitoring skin temperature, heart-rate and movement). The state-of-the-art in real time locating systems is reviewed, and appropriate technologies incorporated into the system. The system, encompassing end-nodes through to backend servers, is presented and practically implemented. Quantitative and qualitative feedback and evaluation is presented from testing in a real hospital environment.

Ammae et al, in their article entitled "Unobtrusive detection of body movements during sleep using $\mathrm{Wi}-\mathrm{Fi}$ received signal strength with model adaptation technique", describe a method for sensing the body movements by measuring changes in Wi-Fi signal strength between two Wi-Fi-enabled devices allowing an unobtrusive way of measuring sleep quality [12]. They adapt a users body movement detection model using other users training data based on using maximum likelihood linear regression (MLLR). This allows them to adapt a user independent movement detection model. They evaluated their method using 60 sessions of real data collected from six participants and achieved high detection accuracy with their user-independent movement detection models.

In the article entitled "A reliable IoT system for Personal Healthcare Devices," Woo et al. [13] focus on the important issue of fault-tolerant health data services. To do so, authors present a fault-tolerant algorithm for the reliable IoT system. In this architecture, gateways can be linked to form a daisy chain for fault tolerance. Moreover, a gateway can store the backup copy of the previous gateway positioned immediately ahead of the gateway in the daisy chain. This approach enables us to recover as many as two gateway faults occurred at the same time.

Rahmani, et al. [14], in the article entitled "Exploiting smart e-Health gateways at the edge of healthcare Internetof-Things: A fog computing approach", present a smart eHealth Gateway at the edge of the network in a Fog-assisted system architecture. This Gateway is capable of offering several features such as local storage, real-time local data processing, embedded data mining, etc. In addition, the proposed Fog-assisted system architecture enables us to tackle many emerging issues in ubiquitous healthcare systems such as mobility, energy efficiency, scalability, and reliability issue. Finally, authors with help of a prototype, describe some of the higher-level features of their proposed gateway such as IoT-based Early Warning Score (EWS) health monitoring.

Finally, Farahani, et al. [2], in their interesting article entitled "Towards fog-driven IoT eHealth: Promises and challenges of IoT in medicine and healthcare," provide a systematic review of IoT architecture for eHealth and mHealth. The authors have thoroughly described the ongoing challenges in healthcare across the globe. In the response, the article proposes to migrate healthcare services from hospital-centered model to person-centered model with the support of IoT. The authors propose a holistic, multi-layer IoT ecosystem for eHealth that is driven by three layers including edge devices, fog nodes, and cloud computing. The article also enumerates the challenges for such IoT ecosystem and proposes potential 

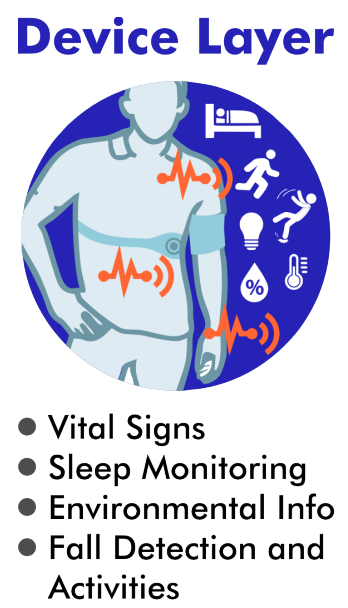

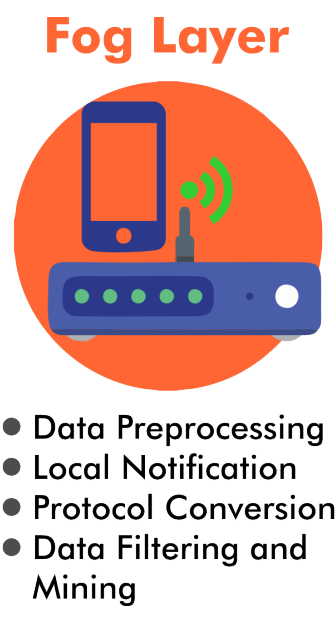

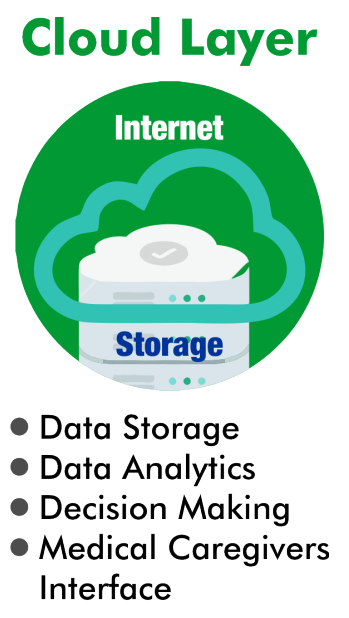

Fig. 2: Architectural Elements of Healthcare IoT Systems.

solutions. The case studies involving smart eyeglasses and smart textiles are presented to demonstrate the capability of the proposed IoT ecosystem for eHealth.

\section{CONCLusions}

Healthcare and technology have always been connected, but that relationship due to the rapid growth of the Internet of Things (IoT) and the popularity of wearable devices has been significantly transformed in recent years. This leads to personalized healthcare, increasing healthcare access and customization the likes of which we have never seen. These advancements, while exciting, should be adopted carefully, as there are still legitimate concerns related to consistency, safety, cost-effectiveness, and more. Many changes need to take place to make this technology viable in the medical field. Most importantly, hardware and software need to be engineered to work together to address novel IoT technologies and their role in the healthcare field. This special issue addressed all important aspects of IoT eHealth technology including smart healthcare wearable sensors, body area sensors, advanced pervasive healthcare systems, and Big Data analytics to provide eHealth services to individuals for healthier lifestyles.

\section{ACKNOWLEDGMENT}

We sincerely hope the reader finds this special issue useful and that it will inspire further research in this very important area of IoT eHealth. We would like to thank all authors who submitted papers to this special issue. Special thanks go to the referees for their time and diligence during the review process and for providing us with high-quality reviews. Finally, we would like to thank Prof. Peter Sloot, Editor-in-Chief of the Elsevier Journal of Future Generation Computer Systems, for offering us the opportunity to edit this Special Issue.

\section{REFERENCES}

[1] GrandViewResearch, available at http://www.grandviewresearch.com/industryanalysis/internet-of-things-iot-healthcare-market.
[2] B. Farahani, F. Firouzi, V. Chang, M. Badaroglu, N. Constant, and K. Mankodiya, "Towards fog-driven iot ehealth: promises and challenges of iot in medicine and healthcare," Future Generation Computer Systems, 2017.

[3] A. Rahmani, P. Liljeberg, J. Preden, and A. Jantsch, Fog Computing in the Internet of Things - Intelligence at the Edge, 1st ed. Springer, Switzerland, 2017.

[4] I. Azimi, A. M. Rahmani, P. Liljeberg, and H. Tenhunen, "Internet of things for remote elderly monitoring: a study from user-centered perspective," J of Ambient Intell Human Comput, vol. 8, no. 2, p. 273289, 2017.

[5] R. Mieronkoski, I. Azimi, A. M. Rahmani, R. Aantaa, V. TerŁvŁ, P. Liljeberg, and S. Salantert, "The internet of things for basic nursing carea scoping review," International Journal of Nursing Studies, vol. 69, pp. $78-90,2017$.

[6] A. M. Rahmani, N. K. Thanigaivelan, T. N. Gia, J. Granados, B. Negash, P. Liljeberg, and H. Tenhunen, "Smart e-health gateway: Bringing intelligence to internet-of-things based ubiquitous healthcare systems," in $C C N C, 2015$, pp. 826-834.

[7] A. M. Rahmani, T. N. Gia, B. Negash, A. Anzanpour, I. Azimi, M. Jiang, and P. Liljeberg, "Exploiting Smart E-Health Gateways at the Edge of Healthcare Internet-of-Things: A Fog Computing Approach," Journal of Future Generation Computer Systems, 2017.

[8] S. R. Moosavi, T. N. Gia, E. Nigussie, A. M. Rahmani, S. Virtanen, H. Tenhunen, and J. Isoaho, "End-to-end security scheme for mobility enabled healthcare internet of things," Future Generation Computer Systems, vol. 64, pp. $108-124,2016$.

[9] B. Negash, A. M. Rahmani, T. Westerlund, P. Liljeberg, and H. Tenhunen, "Lisa 2.0: lightweight internet of things service bus architecture using node centric networking," Journal of Ambient Intelligence and Humanized Computing, vol. 7, no. 3, pp. 305-319, 2016.

[10] J. Pagn, M. Zapater, and J. L. Ayala, "Power transmission and workload balancing policies in ehealth mobile cloud computing scenarios," Future Generation Computer Systems, pp. -, 2017.

[11] T. Adame, A. Bel, A. Carreras, J. Meli-Segu, M. Oliver, and R. Pousa, "Cuidats: An rfidwsn hybrid monitoring system for smart healthcare environments," Future Generation Computer Systems, pp. -, 2017.

[12] O. Ammae, J. Korpela, and T. Maekawa, "Unobtrusive detection of body movements during sleep using wi-fi received signal strength with model adaptation technique," Future Generation Computer Systems, 2017.

[13] M. W. Woo, J. Lee, and K. Park, "A reliable iot system for personal healthcare devices," Future Generation Computer Systems, 2017.

[14] A. M. Rahmani, T. N. Gia, B. Negash, A. Anzanpour, I. Azimi, M. Jiang, and P. Liljeberg, "Exploiting smart e-health gateways at the edge of healthcare internet-of-things: a fog computing approach," Future Generation Computer Systems, 2017. 